\title{
Evaluation of the safety and efficacy of Chinese medicine in people with asymptomatic hyperuricemia: Protocol for a prospective, double- blinded, randomized, placebo controlled clinical trial.
}

Jing-yao Yan

Guangdong Provincial Hospital of Traditional Chinese Medicine

Ying-yan Zhou

Guangdong Provincial Dental Hospital: Guangdong Provincial Stomatological Hospital

Qiao-wen Yang

Guangdong Provincial Hospital of Traditional Chinese Medicine

Jia-qi Wu

Guangdong Provincial Hospital of Traditional Chinese Medicine

Xiao-hong He ( $\nabla$ wdtsxh521@126.com)

Guangdong Provincial Hospital of Traditional Chinese Medicine

\section{Research Article}

Keywords: Chinese medicine, asymptomatic hyperuricemia, double-blinded, randomized, placebo controlled

Posted Date: October 18th, 2021

DOl: https://doi.org/10.21203/rs.3.rs-395566/v1

License: (c) (i) This work is licensed under a Creative Commons Attribution 4.0 International License. Read Full License 


\section{Abstract}

\section{Background}

Hyperuricemia increases the risk of gout and cardiovascular complications. Studys show that Chinese medicine can decrease uric acid through multiple targets, but many of these studys is conducted on animal, because the lack of a consistent prescription and mechanism.So we design this research to study whether Chinese medicine is really effective and which target is essential.

\section{Methods}

This study is a prospective, double-blinded, randomized, placebo controlled clinical trial. From March 2020 to December 2021.30 asymptomatic hyperuricemia people will be recruiting and assigned to either Chinese medicine group or placebo group, each group will have 15 subjects.During the 12-week observation period,there will be 4 times of visits. The decline in the uric acid is the main outcome measure, urinary uric acid,inflammatory biomarkers and other index that may involve in lowing uric acid are the secondary outcome measures.

\section{Discussion}

This study will probe the effect of Chinese medicine treatment on hyperuricemia, and explore possible therapeutic mechanisms. By performing this trial,we hoping to provide evidence and data to support further large clinical studies.

Trial registration:ChiCTR2000038575. Registered on September 24, 2020.

\section{Background}

As living standards rise and dietary habits changes, the prevalence of hyperuricemia has increased[1], especially in China[2]. It had became the second most common metabolic disease after diabetes mellitus and affects 133 persons per 1000 inhabitants in mainland China (the morbidity of men is $19.4 \%$,women is $7.9 \%$ ) [3]. Meanwhile, the prevalence rate is higher in cities than in rural areas, statistics show that the incidence of hyperuricemia in Guangzhou and elderly in Beijing is $13.2 \%$ and $17.6 \%$ respectively[4]. Hyperuricemia may led to GOUT eventually, and accompany augment the risk of hypertension and other cardiovascular disease[5]. Influenced by the Chinese culture of nip in the bud,people with hyperuricemia were seek for early interventions to prevent progressing to GOUT and other disease. Nonetheless, they do not prefer accept uric-acid-lowing drugs, as the hyperuricemia is considered as "not ill". Thus, many people quest for Chinese medicine treatments.

Some animal studies have shown that Chinese medicine can lower uric acid levels,relief symptoms and reduce the frequency of Gout attacks[6-12]. The mechanism of Chinese medicine is complex and not clear yet. Some study indicates Chinese medicine may promote the uric acid excretion[6-8]; Other researchs has shown that, Chinese medicine can modulate the levels of inflammatory cytokines[9-12]; 
Evidences also suggest that Chinese medicine inhibit the synthesis of uric acid[13, 14]. So our trial will test subjects' capacity of uric acid excretion and inflammation index.Recently, there are trials support the difference in intestinal microecology between hyperuricemia people and healthy people[15, 16]. Rats experiments showed Chinese medicine has a certain influence on the composition of intestinal flora[17, 18]. Therefore intestinal microecology before or after Chinese medicine treatment is our another interest potential target.

How to evaluate the efficacy of Chinese medicine treatments and develop effective treatment for asymptomatic patients with hyperuricemia is difficult, for Chinese medicine treats patients based on syndrome differentiation rather than disease differentiation, thus the recipes for Chinese medicine for people may vary in composition. So it is essential to establish syndrome standards. Due to the lack of relevant literatures and researchs, we gathered experts of Chinese medicine classic, by consulting Chinese medicine classics and combining their clinical experience, to reach a consensus, that for people with hyperuricemia in Guangdong province, dampness syndrome is the main syndrome.

We designed questionnaires, adopted 11 people with hyperuricemia by electronic questionnaire system. The result showed that all the 11 cases were consistent with the clinical manifestations of dampness syndrome. 8 of which had the manifestations of cold dampness syndrome, 3 showed the manifestations of hot dampness syndrome. The minimum of dampness score is 43 , and maximum is 104 , the average is $70.09 \pm 17.56$. Summarize the performance of the 11 cases, $54.5 \%$ of which has superficial syndrome. Based on clinical experience, combined the concept of Chinese medicine that "the beginning of disease is in the grain of skin and the texture of the subcutaneous flesh", guided by the Chinese medicine theory of preventive treatment of diseases, combined with the opinions of many Chinese medicine classic experts, put forward the treatment viewpoint of "treating metabolic diseases at the early stage by skin". Choose the "tuckahoe alisma soup"which is specific to Taiyang YangMing Taiyin combination diseases as the main prescription for the cold dampness population, in the ingredient of the soup, tuckahoe, atractylodes can dissolve the wet inside,cassia twig and ginger work on the surface, treat the disease out and in simultaneously, urge the wet out with alisma; For people with hot dampness, the main prescription is "Scutellaria and ginger pinellia soup".

\section{Methods And Design}

\section{Study objective}

The objectives of the experiment is to study the efficacy and safety data of Chinese medicine therapy for dampness syndrome of hyperuricemia; And to screen possible related intuitive small molecules/microbial material basis.

\section{Study design}

This is a 12 weeks, single-center prospective, double-blinded, randomized, placebo controlled clinical trial. We used the SPIRIT reporting guidelines to perfecting this protocol[19]. From March 2020 to December 
2021. Incorporate 30 asymptomatic hyperuricemia subjects, which will be randomly 1:1 assigned to receive Chinese medicine or placebo.

\section{Sample size}

This study will enroll 30 subjects. Further large-scale RCT trial will estimate the size of sample according to the results of the main outcome indicators obtained from this study, use the sample content estimation formula for comparing the two means to calculate the sample content, $\alpha=0.025, \beta=0.2$, The sample size ratio of test group and control group is 1 , The lost follow-up rate is set according to the pre-experiment lost follow-up rate.

\section{Recruitment}

Most asymptomatic patients with hyperuricemia do not seek for medical attention, so we will recruit subjects through recruitment advertisements. The advertisements will be placed in the hospital building, and be broad through the hospital official account.

\section{Study setting}

Guangdong provincial hospital of Chinese medicine will be the only study setting.

\section{The study's key problem}

Dampness is involved with Hyperuricemia developing. The key of prevent gout, cardiovascular disease and uratic nephropathy is control the serum uric acid level. Weather Chinese medicine can improve the outcomes of hyperuricemia population, access the goal of early prevent disease?

\section{Eligibility criteria}

Inclusion criteria:

1. Aged between $18-75$ years(including 18 years and 75 years;

2. Meet the diagnosis of asymptomatic hyperuricemia;

3. Meet the dampness syndrome inclusion criteria;

4. Sign the informed consent.

Exclusion criteria:

1) Secondary hyperuricemia,for instance,secondary to malignancy,hemopathy $₫$ nephropathy $\llbracket$ chronic intoxication;

2)History of alcoholism without abstinence in recent 3 months;

3) Had one or more attacks of gout arthritis in the past; 
4)Received urate-lowing drugs or drugs that may increase serum uric acid in recent 4 weeks: (1) antituberculotic:Pyrazinamide/ethambutanol/isoniazid, (2) low dose aspirin, (3) Loop diuretics and thiazide diuretics and blood pressure medications containing diuretics, (4) niacin, (5) large dosage of vitamin C, (6) chemotherapeutic drugs, (7) cyclosporin;

5) Pregnancy and lactation population, or has possibility of conception but failure to use effective contraception;

6) Alanine aminotransferase or aspartate aminotransferase or creatinine 2 times or more than the normal value;

7) Combined with serious organic lesions, mental disorders or other reasons can not cooperate with treatment; 8) Allergic or ineffective to the test drug ingredients.

\section{Randomization and allocation}

Adopting simple block group randomization, the allocation ratio will be 1:1. Use SAS9.2 PROC PLAN to carry out procedure coding and random sequence generation by methodology team.

The random distribution results will released through the "Interactive network response random distribution System for Clinical Research in Guangdong provincial hospital of Chinese medicine. The random assignment table in the random Center will be kept strictly confidential until the end of the study.

After the patients are screened as qualified subjects and given informed consent, the clinicians will log on the Internet to apply for the randomization system to obtain the randomization results. Physicians execute the results according to random grouping, and print and paste them on the corresponding position of CRF.

\section{Blind}

Adopt double-blinded, placebo controlled design, both the investigators and the subjects are blinded.All data will be evaluated and statistically processed by a third-party after the clinical research.

The blindness should be broken if there is a serious adverse event that may be related to the experimental drug, so we can know which group the subject was enrolled in to decide the rescue plan. The blindness should be broken in the presence of principal investigator and funders. The time, reason, procedure of the unblinding and the the researchers involved in the unblinding should be recorded. After the blindness was broken, the subject will be terminated from the study, and the experimental data will be included in the safety analysis data set, and the subject was given timely treatment and follow-up.

\section{Comparators}

Many Chinese medicine studies set Chinese medicine plus western medicine as the experimental group and soly modern medicine as the control group. This may lead to bias. So we use placebo control group 
as the comparators.

\section{Chinese medicine syndrome subgroups}

All enrolled subjects will be assigned to subgroups: cold-dampness disturbing spleen and damp-heat retention spleen. The definition of which is to draw up with refer to "The guiding Principles for clinical Research of New Chinese medicines (trial)" in 2002, which was edited by Zheng Xiaoyu[20].

\section{Interventions}

Subjects will be given therapeutic agent (Chinese medicine) or placebo.

Chinese medicine intervention group: 1) Cold-dampness disturbing spleen: Tuckahoe alisma soup, one dose a day, warm take after meal. 2) Damp-heat retention spleen: Scutellaria and ginger pinellia soup, one dose a day, warm take after meal.

Chinese medicine placebo internal administration: the size, appearance, package and the the administration method are the same as the therapeutic drugs. During the trial, the investigators will monitor dietary and exercise conditions of subjects everyday.

\section{Eliminate and Termination Criteria}

Eliminate criteria: 1) Serious violation of exclusion standards after enrollment; 2)Not completing the experimental drug administration during the experimental period as required after enrollment; 3 ) Incomplete one month follow up, no test records were available for evaluation.

Termination Criteria: 1)Have a gout attack; 2) sever adverse events; 3) Condition deteriorated and required urgent medical attention; 4) The researcher recommended terminate test because of the safety of the subjects. 5)People unwilling to continue to participate in this study for personal reasons.

We will keep in touch with the subjects who drop out to observe any subsequent adverse event and collect subsequent data.

\section{Strategies to improve adherence to interventions}

The compliance of investigators and subjects is essential factors of clinical research process and clinical effect. To ensure compliance, investigators or the designated representative must explain the detailed information about the clinical trail to the subjects, and to obtain informed consent after sufficient and detailed explanation.

The compliance evaluation of the subjects is mainly judged from the status recorded by the subject's log card and the status questioned by the researchers.

Plans to promote participant retention and complete follow-up 
First, the investigators will keep in touch with the subjects, regular telephone follow-ups and reminders for follow-up visits will be implement. Second, our research system will prompt the prescription a day in advance. Third, the subjects will receive free tests. Forth, transportation subsidies will be given after each follow-up visit to encourage subjects to remain engaged and complete follow-up surveys.

\section{Outcome measures}

\section{Primary outcome measure}

The reduction of serum uric acid.

\section{Secondary outcome measures}

1)Chinese syndrome score, measurement of the channels and collaterals, tongue diagnosis evaluation; 2 ) Glomerular filtration rate, Urinary $\beta 2$ microglobulin, uric acid excretion fraction; 3) Incidence rate of complications, such as gout, hyperlipemia, cardiovascular events, diabetes, etc; 4) Ultrasound of double ankles and the first metatarsophalangeal joints[21]; 5) Evaluation index of metabolite: blood, urine metabolite. 6) Inflammation index: hsCRP, TNF-a, IL-6, IL-8; 7) Intestinal microecology( Note: This index is tested by setting a separate subject).

\section{Security indicators}

1)Liver function(ALT, AST), renal function(Urea, Cr); 2) Blood routine, urine routine, stool routine + occult blood; 3 ) lectrocardiogram, ultrasound of the urinary system.

\section{Data collection and analysis}

Within 12 weeks course there will be 4 times of visits: 0 week, 4 weeks \pm 3 days, 12 weeks \pm 5 days, and the date collection and the filling of the CRF will be performed by two research assistants who have no idea about the subjects grouping. Adopt EPIdata3.1 database software package to establish a database for data entry, use double input data for data entry.

The clinical trial monitors check the quality of data entry regularly. The checked database is then checked by logic inspection and the original laboratory documents of outliers, and the finally checked database is converted into the DATABASE in the format of SPSS statistical software package and locked for statistical analysis.

\section{Statistical analysis}

All statistical tests are two-sided, $\mathrm{a}=0.05$ will be found statistically.

We will use t-test (or wilcoxon matched-pairs signed-ranks test if the data is non-normal distribution with uneven variances) to compare the decrease in the uric acid between two groups. 
For secondary outcomes, the t-test and Wilcoxon test will be conducted to compare the differences in measurement data (such as index's mean and standard deviation, median) between the groups. The Repeated measures analysis of variance will be used to compare multiple measures of the scale and symptom rating among groups. The Chi-square test (or Fisher's Exact test) and $3 \times \mathrm{C}$ table will be utilized to determine the differences in the proportion, frequency, total effective rate of the indices in Chinese medicine group and placebo group.

To analysis the influence factors of curative effect, the nonconditional logistic regression analysis will be performed.For missing data from long-term follow-up, the last carry-over method (LOCF) will be used.

\section{Safety analysis}

Safety data is consist of adverse events and clinical laboratory tests. We will describe specific manifestations of adverse reactions and compare the incidence of adverse events and sever adverse events between two groups. The changes of laboratory test results before and after the test, abnormal changes and their relationship with Chinese medicine will be analysis.

\section{Compliance analysis}

A descriptive analysis are will be exploit to analyze compliance among the subgroups included in the study, and between the two groups for baseline of abscission cases.

\section{Adverse events Management}

The type, degree, occurrence time, duration, treatment measures, treatment process and follow-up results of adverse events occurred during the trial will be recorded in the case report form, and the correlation between the adverse events and the experimental drugs will be evaluated on the basis of comprehensive consideration of the complications and combined use of drugs, which will also be recorded in detail by the physician.

For non-serious adverse event, the observation physician may decide whether to suspend observation based on the disease condition. Cases that withdrawal drugs due to adverse event will be followed up and reexaminate the lab security indicators.

For serious adverse event, drug taking will be stopped immediately. Regardless of whether it is related to the experimental drug,the investigators should report to the Ethics Committee of Guangdong province hospital of Chinese medicine within 24 hours after its occurrence.

If a serious adverse event occurs during the course of the study, the research team and the hospital will be responsible for subsequent treatment and financial compensation.

\section{Protocol amendments}


If a protocol change is required, the risk to the subject should be fully assessed and reviewed by the Ethics Committee.Inform all members after the new scheme has been approved by email and face-to-face meeting.

\section{Confidentiality}

Subjects have independent codes and only enter codes when uploading test data. Encryption system will be utilized to upload and store datas. The final statistically process will be administrated by a third-party.

\section{Discussion}

Our aim is through carrying out early intervention of Chinese medicine in the stage of asymptomatic hyperuricemia with"not ill", by controlling serum uric acid, thus can decrease the probability of asymptomatic hyperuricemia develop gout and other risk factors of cardiovascular and cerebrovascular disease, prevent and treat disease in early stage.

This research based on previous years of clinical experience and cross-sectional investigation and analysis,plan to carry out pre-research,to proceed study aimed at hyperuricemia people with dampness syndrome in Gouangdong, on the basis of the pre-research, the sample size will be expanded and the randomized controlled double-simulation clinical trial will be conducted to obtain high-level evidencebased evidence of Chinese medicine in the treatment of hyperuricemia with dampness syndrome, thus to form an effective early Chinese medicine intervention plan for the population with hyperuricemia with dampness syndrome.

\section{Trial Status}

This trial information is available on chictr.org.cn, protocol version 1.4, November 30,2020 . The study was funded on February 25, 2020. Our trial enrolment period is from March to August 2021.

\section{Declarations}

\section{Ethics approval and consent to participate}

This study was approved by the Ethics Committee of Guangdong provincial hospital of Chinese medicine(BF2020-172-01) .

All the subjects involved in the research will be informed the objectives, duration, treatment, possible risks,rights and interests, etc. Investigators or the designated representative will obtain the written, informed consent to participated from all participants. The study consent process includes permission to collect intestinal flora specimens for additional trials.

\section{Consent for publication}

Not applicable. 
Availability of data and materials

The data will not be shared, because this is a preliminary experiments. The research unit will have access to the final trial data set.

\section{Competing interests}

The authors declare that they do have no competing interests. The study funder has no role in the design, execution, analyses, interpretation of data, or decision to submit results for this study.

\section{Funding}

This trial is financially supported by the Project of State Key Laboratory of Dampness Syndrome of Chinese Medicine.

\section{Author information}

\section{Affiliations}

State Key Laboratory of Dampness Syndrome of Chinese Medicine, The Second Affiliated Hospital of Guangzhou University of Chinese Medicine. 111 Dade Road, Yuexiu District, Guangzhou, Guangdong 510000, China.

Department of Rheumatology, Guangdong provincial hospital of Chinese Medicine, No. 55 of Neihuanxi Road, Higher Education Mega Center, Guangzhou 510006, China.

Yan Jing-yao,Zhou Ying-yan,Yang Qiao-wen,Wu Jia-qi,He Xiao-hong.

\section{Contributions}

HXH, YJY, ZYY, YQW, WJQ received the research funding. HXH conceived the study, designed the study protocol and ethical approval. ZYY drafted the manuscript. YJY, YQW, WJQ participated in the design of the protocol and in charge of clinical case collection and observation. All authors read and approved the final manuscript.

\section{Corresponding author}

Correspondence to He Xiao-hong (wdtsxh521@126. com).

\section{Acknowledgements}

We thanks all the participating subjects for their trust and coordinate. This trial is supported by the Project of State Key Laboratory of Dampness Syndrome of Chinese Medicine.

\section{Dissemination policy}


Regardless of the size and direction of the validity of the test results, they will be published.

\section{References}

1. Pascart T, Lioté F. Gout:state of the art after a decade of development. Rheumatology. 2019;58(1):27-44.

2. Li Lin Z. Xiao-xia, Dai Yu, et al. Chinese multi-disciplinary consensus on the diagnosis and treatment of hyperuricemia and its related diseases. Chinese Journal of Internal Medicine. 2017;56(03):23548.

3. Liu R, Han C, Wu D, et al. Prevalence of Hyperuricemia and Gout in Mainland China from 2000 to 2014: A Systematic Review and Meta-Analysis. BioMed research international, 2015, 2015: 762820.

4. Wang Dan-chen. Yin Yi-cong, Yu Song-lin. Survey for variation of prevalence of hyperuricemia and its correlations with blood glucose and lipid in healthy adults receiv-ing physical examination at Peking Union Medical College Hospital from 2012 to 2017. Chinese Journal of Clinical Laboratory Science. 2018;36(06):462-6.

5. Abeles AM, Pillinger MH. Gout and cardiovascular disease: crystallized confusion. Curr Opin Rheumatol. 2019;31(2):118-24.

6. Xing WANG, Ning XUE, Hong-lei LI, et al. Study on mechanism of Fangji Huangqi Decoction on hypouricemic effect and renal protection in hyperuricemia mice. China Journal of Chinese Materia Medica. 2020;45(21):5248-55.

7. FANG C, CHEN L Y, HE M Z, et al. Molecular mechanistic insight into the anti-hyperuricemic effect of Eucommia ulmoides in mice and rats[J]. Pharm Biol. 2019;57(1):112-9.

8. Jin-xiang ZENG, Bing-bing XU, Juan WANG, et al. Hypouricemic effects of acteoside and isoacteoside from Plantaginis Semen on mice with acute hyperuricemia and their possible mechanisms. Chinese Traditional Patent Medicine. 2016;38(07):1449-54.

9. Xiaoxi ZHANG, Mengyu XING, Xinyu ZHAO,et al. Effect and Mechanism of Modified Sanmiao Pill in the Treatment of Gouty Arthritis Through NLRP3 Inflammasome Axis and NF- KB Signaling Pathway. Journal of Zhejiang Chinese Medical University. 2019;43(10):1130-7.

10. Yuan-yuan ZHOU. LI Zhong-nan. Effect of Biling Prescription on PGE2 and COX-2 Levels in Diabetic Gout Model Rats. JOURNAL OF JIANGXI UNIVERSITY OF TCM, 2020, 32(1): pp. 83-6.

11. Xue-fang YANG, Yong-chang WANG, Yong-dong ZHANG, et al. Effect of Different Extracts from Modified Siwu Siteng Decoction on Acute Gouty Arthritis in Rats. Chinese Journal of Experimental Traditional Medical Formulae. 2014;20(7):147-51.

12. Yan LAN, Zhanyuan LIU, Lifu XIE. Influence of Xuanbi decoction on serum high-sensitivity C-reactive protein(hs-CRP)and matrix metalloproteinase-3ロMMP-30levels of patients with acute gouty arthritis. China Modern Doctor. 2014;52(17):76-8.

13. PING Fan TAN, Chang YAN, Zhi zhao, et al. Experimental Study of Bixie Chubi Tang in Treating Gout. Chinese Journal of Experimental Traditional Medical Formulae. 2015;21(9):129-32. 
14. Min ZHOU, Xiao-ming LUO, Wei ZHANG, et al. The Influences of Biqing Capsule on The Uric Acid Metabolism in Chicken Model of Hyperuricemia[J]. Chinese Journal of Experimental Traditional Medical Formulae. 2006;12(12):35-6.

15. Guo Z, Zhang J, Wang Z, et al. Intestinal microbiota distinguish gout patients from healthy humans. Sci Rep. 2016;6:20602.

16. Xing SC, Meng DM, Chen Y, et al. Study on the diversity of Bacteroides and Clostridium in patients with primary gout. Cell Biochem Biophys. 2015;71(2):707-15.

17. Xing LI, Fu-jiang CHU, Shi-lin JIANG, et al. Preliminary study on effect of Phellinus igniarius ethanol extract on serum uric acid metabolism and gut microbiome in rats. China Journal of Chinese Materia Medica. 2021;46(01):177-82.

18. Song Ke-yu, Jiang Zhen-you, Yan Qun-chao, et al. Experimental study on the effect of Radixcodonopsis and Poria on intestinal flora in mice. The Chinese Journal of Clinical Pharmacology, 2011, 27(02): 142-145.

19. Chan A-W, Tetzlaff JM, Gøtzsche PC, Altman DG, Mann H, Berlin J, Dickersin K, Hróbjartsson A, Schulz KF, Parulekar WR, Krleža-Jerić K, Laupacis A, Moher D. SPIRIT 2013 Explanation and Elaboration: Guidance for protocols of clinical trials. BMJ. 2013;346:e7586.

20. Zheng Xiao-yu,etal.The guiding Principles for clinical Research of New Chinese medicines (trial).China medical science press.2002.

21. Richette P, Doherty M, Pascual E,etal. 2018 updated European League Against Rheumatism evidencebased recommendations for the diagnosis of gout.Ann Rheum Dis.2020 Jan;79(1):31-38.

\section{Figures}




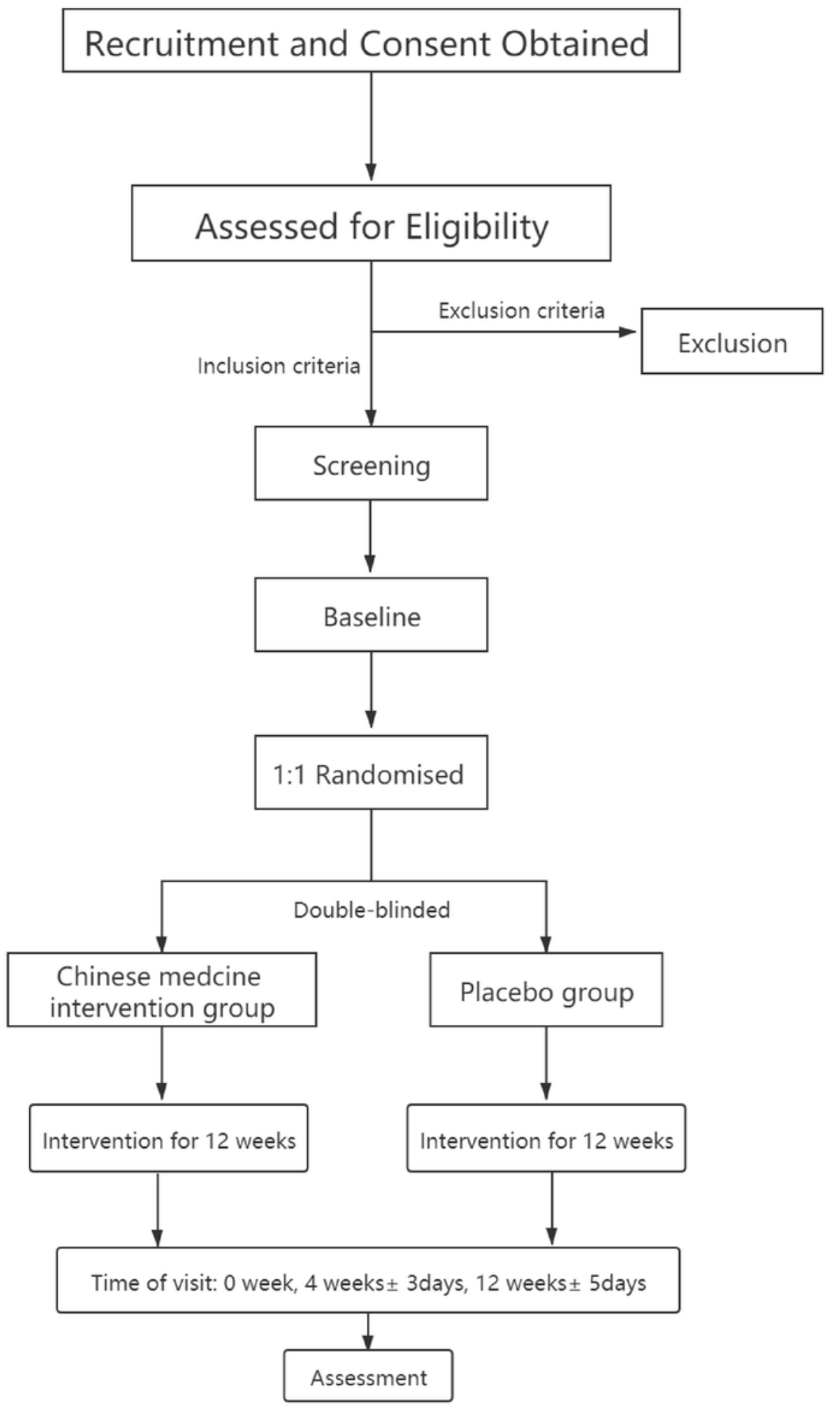

Figure 1

Flow diagram for the study 


\begin{tabular}{|c|c|c|c|c|}
\hline \multirow[b]{2}{*}{ Timepoint } & \multicolumn{4}{|c|}{ Study period } \\
\hline & Visit 0 & $\begin{array}{l}\text { Visit } 1 \\
\text { (0) }\end{array}$ & $\begin{array}{c}\text { Visit } 2 \\
\text { (4 weeks } \pm \text { 3days) }\end{array}$ & $\begin{array}{c}\text { Visit } 3 \\
\text { (12 weeks } \pm \text { ddays) }\end{array}$ \\
\hline Activity/assessment & $\begin{array}{l}\text { Screening and } \\
\text { consent }\end{array}$ & Allocation & Post-allocation & Close-out \\
\hline \multicolumn{5}{|l|}{ Enroll } \\
\hline Eligibility screening & $\mathrm{v}$ & & & \\
\hline Informed consent & $v$ & & & \\
\hline Allocation & & $v$ & & \\
\hline \multicolumn{5}{|l|}{ Intervention } \\
\hline $\begin{array}{l}\text { Chinese medicine intervention } \\
\text { group }\end{array}$ & & v & V & \\
\hline Placebo group & & $v$ & $\mathrm{v}$ & \\
\hline \multicolumn{5}{|l|}{ Assessment } \\
\hline Underlying disease & $\mathrm{v}$ & & & \\
\hline Medication & $\mathrm{V}$ & V & $\sqrt{ }$ & V \\
\hline Drinking Status & $v$ & $v$ & $v$ & $v$ \\
\hline Blood uric acid levels & & $v$ & $v$ & $v$ \\
\hline Chinese syndrome score & & $v$ & $v$ & $v$ \\
\hline Renal excretion index & & $\sqrt{ }$ & $\sqrt{ }$ & v \\
\hline Complications & $\mathrm{v}$ & $\mathrm{v}$ & $v$ & $v$ \\
\hline Joints ultrasound & & $\mathrm{v}$ & $\mathrm{v}$ & $v$ \\
\hline Metabolite evaluation index & & $\mathrm{v}$ & $\mathrm{v}$ & $v$ \\
\hline Inflammation index & & $\mathrm{v}$ & $\mathrm{v}$ & $\mathrm{v}$ \\
\hline Intestinal microecology & & v & $\sqrt{ }$ & v \\
\hline Security indicators & & $\mathrm{v}$ & $\mathrm{v}$ & $v$ \\
\hline Diet and exercise & $\mathrm{v}$ & $\mathrm{v}$ & $\mathrm{v}$ & $\mathrm{v}$ \\
\hline
\end{tabular}

\section{Figure 2}

Participant timeline

\section{Supplementary Files}

This is a list of supplementary files associated with this preprint. Click to download.

- Modelinformedconsentform.docx

- SPIRIT.docx 
Page 15/15 